\title{
Perfiles de formación bibliotecológica en algunas universidades de América del Sur
}

Library science education profiles in some Latin America universities

\author{
María Teresa MúNERA TORRES \\ Universidad de Antioquia, Calle 67 No. 53-108, Ciudad Universitaria, Bloque 12 Tercer Piso, mmuner@bibliotecologia.udea.edu.co
}

\begin{abstract}
Resumen
Se presenta un panorama general sobre los perfiles de formación bibliotecológica en algunas universidades de América del Sur, a la luz de la investigación "Factores de calidad y competitividad en la educación superior: la educación bibliotecológica en América Latina", liderada por la Universidad Nacional Autónoma de México, con el apoyo de la Universidad Autónoma de San Luis de Potosí, la Universidad de Costa Rica y la Universidad de Antioquia.
\end{abstract}

Palabras clave: Bibliotecología. Estudios de grado. Perfiles profesionales. América del Sur.

\section{Introducción}

A la luz de la investigación "Factores de calidad y competitividad en la educación superior: la educación bibliotecológica en América Latina", proyecto desarrollado por la Universidad Nacional Autónoma de México, bajo la coordinación de la Doctora Lina Escalona Ríos y con el aporte de la Universidad Autónoma de San Luis de Potosí, la Universidad de Costa Rica y la Universidad de Antioquia, se pretende con esta comunicación, presentar una reflexión acerca de los perfiles de formación bibliotecológica en algunas universidades de América del Sur, región que fue el objeto de estudio específico de la Escuela Interamericana de Bibliotecología de la Universidad de Antioquia.

Para comenzar se hace una reflexión sobre el interés por el estudio de los perfiles de formación bibliotecológica, así como los aspectos más importantes de los que se ofertan en algunas universidades de América del Sur y culminar con unas conclusiones sobre el tema expuesto.

\section{Interés por el estudio de los perfiles de formación bibliotecológica}

En el ámbito de la investigación, se han generado estudios sobre las tendencias de formación en Bibliotecología, tanto por expertos en el área, como por grupos interesados en esta línea de investigación. Ejemplo de ello es el Euroreferencial en información y documentación (ECIA,

\begin{abstract}
An overview of librarianship training profiles in some South American universities is presented, as a result of the project "Factors of quality and competitiveness in higher education: library education in Latin America", which is led by the National Autonomous University of Mexico, with the support from the Autonomous University of San Luis Potosi, the University of Costa Rica and the University of Antioquia.
\end{abstract}

Keywords: Library science. Pregraduate studies. Professional profiles. South America.

2004), que brinda una pauta frente a las competencias y aptitudes de los profesionales europeos de información y documentación. También el Instituto de Investigaciones Bibliotecológicas (IIBI) de la UNAM de México, cuenta con un grupo permanente de investigación en educación bibliotecológica, conformado por docentes e investigadores de Iberoamérica.

Actualmente se está culminando la investigación "Factores de calidad y competitividad en la educación superior: la educación bibliotecológica en América Latina". En este trabajo se enfocaron tres regiones: México, Centro América y Sur América. La región de México fue abordada por las Universidades Nacional Autónoma de México y Autónoma de San Luis de Potosí; la región de Centro América, por la Universidad de Costa Rica; y la de Sur América por la Universidad de Antioquia. En esta investigación se desarrolló una metodología que combinó tanto la investigación documental como la de campo. Por tanto, se estudiaron escuelas de bibliotecología de las regiones anteriormente mencionadas, desde la perspectiva no solo de su historia, perfiles de formación y estructuras curriculares, sino también desde la aplicación de procesos de evaluación y de aseguramiento de la calidad. Sin embargo, en esta reflexión se abordará solo lo atinente a los perfiles de formación bibliotecológica en la Región de América del Sur.

Las tendencias en los procesos de formación bibliotecológica se constituyen en temas de 
interés para los investigadores de la bibliotecología contemporánea, debido a la introducción de paradigmas e innovaciones que se manifiestan desde diversos ámbitos. Es así como desde la segunda mitad del siglo XX, el avance tecnológico y las nuevas formas de gestión de la información y del conocimiento, empiezan a aparecer en escena, con la adopción de cambios en el desempeño profesional bibliotecológico, lo que motiva procesos de revisión y actualización de los perfiles y propuestas de educación en esta disciplina.

Fenómenos como el de la globalización estimulan el origen de políticas y lineamientos a nivel mundial desde la dimensión económica, productiva, laboral, profesional, social, política; y, a su vez, marcan la pauta a seguir por parte de los gobiernos, en cuanto al tipo de profesionales que se debe formar, según los intereses y demandas de los grupos que lideran la economía mundial. Sobre el particular, Lina Escalona (2008, p. 8-9) precisa:

Esta globalización ha afectado a la educación bibliotecológica, fundamentalmente en dos áreas: A nivel externo, en el mercado de trabajo, la forma en que se desarrollan las actividades profesionales y no profesionales del bibliotecólogo han cambiado y han surgido nuevas actividades para las que el bibliotecario "tradicional" no estaba preparado, por ejemplo el diseño y venta de servicios de información o la inclusión en los sistemas de información digitales y/o virtuales. A nivel interno, el currículo se torna cada vez más complejo ya que se debe formar a los estudiantes para enfrentar los retos de la sociedad globalizada, por lo que además de los conocimientos teórico prácticos disciplinarios, también se les debe enseñar el manejo de las nuevas tecnologías aplicadas a sus actividades y deben manejar varios idiomas, de modo que puedan demostrar su competitividad a nivel nacional e internacional.

Al respecto, otras investigadoras (Martínez Rider y Chacón Escobar, 2010, p. 44) señalan como

La globalización y el modelo neoliberal han tenido consecuencias en la educación superior y debemos replantear las prácticas profesionales y las concepciones disciplinares o profesionales. Simultáneamente la Universidad pública trata de rescatar su proyecto social frente a una realidad diversa y concreta que se inserta dentro de la desregulación de la economía de mercado y tiene efectos variados en América Latina.

El impacto del desarrollo científico y tecnológico ha estimulado la asimilación de nuevas tendencias en los procesos de desarrollo económico y social de diversos sectores de la sociedad y de manera particular en las dinámicas educativas, razón por la cual las instituciones de educación superior adoptan como parte de su labor, la revisión de las propuestas de formación profesional, a la luz de los adelantos y desarrollos que se plantean a la sociedad del siglo XXI. Karla Rodríguez Salas (2009, p. 379) señala:

Tendencias como el desarrollo económico y social, caracterizado por la incorporación de un nuevo factor productivo basado en el conocimiento y en el manejo adecuado de la información, o las nuevas tecnologías de la información y comunicación, nos piden una nueva concepción del perfil profesional, centro del proceso de enseñanza aprendizaje constituido por el estudiante y el alcance global de la actividad humana, lo cual hace que las universidades asuman la actualización continua de los contenidos de sus planes de estudio empleando enfoques multi e interdisciplinarios.

Ante este panorama de transformaciones permanentes en el ámbito mundial, resulta pertinente apreciar la dinámica que presentan los perfiles de formación bibliotecológica y de manera especial en los países de América del Sur, con el propósito de asimilar las tendencias que se manifiestan en esta región.

\section{Perfiles de formación}

Partiendo de concebir la definición de perfil profesional como (Hawes y Corvalán, 2006, p. 9):

El conjunto de rasgos y capacidades que, certificadas apropiadamente por quien tiene la competencia jurídica para ello, permiten que alguien sea reconocido por la sociedad, como 'tal profesional, pudiéndosele encomendar tareas para las que se le supone capacitado y competente.

Resulta interesante apreciar, de manera general, los aspectos más sobresalientes de los perfiles de formación bibliotecológica de 29 universidades de países como Argentina, Bolivia, Brasil, Chile, Colombia, Ecuador, Paraguay, Perú, Uruguay y Venezuela (Tabla I, en apéndice).

Para hacer una aproximación general a los aspectos más relevantes de los perfiles de formación bibliotecológica de los países anteriormente mencionados, se tienen en cuenta aspectos como: títulos que se otorgan, tiempo de duración de los estudios, tendencias de formación de los programas y campos de desempeño más destacados.

\subsection{Títulos que se otorgan}

Al abordar las propuestas de formación bibliotecológica de la región objeto de interés en esta comunicación, se aprecia diversidad de nombres en las titulaciones que se otorgan a quienes completan su formación profesional en esta área, debido entre otras causas, a fenómenos 
que han generado una nueva dimensión de la bibliotecología contemporánea.

Sobre el particular se destaca el hecho de que el título de Bibliotecólogo y Bibliotecónomo prevalece, no obstante, la introducción de nuevas formas de desempeño profesional. Se observa también la introducción de propuestas de titulación tales como las de profesional en Ciencia de la Información y Ciencias de la Información, conceptos que surgen, como resultado del advenimiento de la Sociedad de la Información y el Conocimiento, así como de la vinculación de las Tecnologías de la Información y Comunicación en los campos de desempeño bibliotecológicos. En la (Tabla II) se presenta, la relación de algunas de las titulaciones de la formación bibliotecológica en América del Sur y el número de universidades que las utiliza, de acuerdo con las instituciones que han sido objeto de estudio.

\begin{tabular}{|c|c|c|}
\hline \# & Denominaciones & $N^{\circ}$ \\
\hline 1 & Archivología y Bibliotecología & 1 \\
\hline 2 & $\begin{array}{l}\text { Bachiller Superior en Bibliotecología y Ciencias de la } \\
\text { Información }\end{array}$ & 1 \\
\hline 3 & Bibliotecología & 4 \\
\hline 4 & Bibliotecología y Archivología & 1 \\
\hline 5 & Bibliotecología y Ciencias de la Información & 2 \\
\hline 6 & Biblioteconomía & 6 \\
\hline 7 & Biblioteconomía y Documentación & 3 \\
\hline 8 & Ciencia de la Información & 1 \\
\hline 9 & Ciencia de la Información-Bibliotecología & 1 \\
\hline 10 & Ciencia de la Información y Bibliotecología & 1 \\
\hline 11 & Diplomatura en Bibliotecología & 1 \\
\hline 12 & Gestión de Información, Bibliotecas y Archivos & 1 \\
\hline 13 & Historia con mención en Archivología y Bibliotecología & 1 \\
\hline 14 & Licenciatura en Bibliotecología & 1 \\
\hline 15 & $\begin{array}{l}\text { Licenciatura en Bibliotecología y Ciencia de la } \\
\text { Información }\end{array}$ & 1 \\
\hline 16 & $\begin{array}{l}\text { Licenciatura en Bibliotecología y Ciencias de la } \\
\text { Información }\end{array}$ & 2 \\
\hline 17 & Licenciatura en Bibliotecología y Documentación & 1 \\
\hline 18 & Licenciatura en Ciencias de la Información & 2 \\
\hline 19 & $\begin{array}{l}\text { Profesorado en Bibliotecología y Ciencia de la } \\
\text { Información }\end{array}$ & 1 \\
\hline 20 & $\begin{array}{l}\text { Profesorado en Bibliotecología y Ciencias de la } \\
\text { Información }\end{array}$ & 1 \\
\hline 21 & Sistemas de Información y Documentación & 1 \\
\hline 22 & $\begin{array}{l}\text { Técnico Superior en Bibliotecología y Ciencias de la } \\
\text { Información }\end{array}$ & 1 \\
\hline
\end{tabular}

Tabla II. Algunas denominaciones de la formación bibliotecológica en América del Sur

\subsection{Tiempo de duración de los estudios}

Con relación a este aspecto, se observa una mayoría de programas académicos que oscila en general entre los 4 y 5 años; y en algunos casos, 7 años, como se manifiesta en algunas universidades brasileñas, cuyos planes de estudio registran una duración entre 4 y 7 años.

También se logra apreciar ciclos de formación de menor duración en programas de tres años, que se corresponden con niveles técnicos que no conducen a titulación profesional. No obstante, aparecen algunos de estos niveles de formación, con denominaciones de carácter profesional. Es el caso de la Universidad Nacional de Córdoba en Argentina, que oferta el programa de Bibliotecología, con una duración de 3 años. En la Tabla III se indica la relación entre la duración de cada oferta académica de educación bibliotecológica y las universidades responsables del desarrollo de cada una.

\begin{tabular}{|c|c|c|c|}
\hline Años & \# & Nombres de los programas & Universidad \\
\hline \multirow[t]{17}{*}{5} & 1 & $\begin{array}{l}\text { Licenciatura en Bibliotecología y } \\
\text { Ciencia de la Información }\end{array}$ & (UBA, ARG) \\
\hline & 2 & $\begin{array}{l}\text { Profesorado en Bibliotecología y } \\
\text { Ciencia de la Información }\end{array}$ & (UBA, ARG) \\
\hline & 3 & $\begin{array}{l}\text { Licenciatura en Bibliotecología y } \\
\text { Documentación }\end{array}$ & (UNC, ARG) \\
\hline & 4 & $\begin{array}{l}\text { Licenciatura en Ciencias de la } \\
\text { Información }\end{array}$ & (UNN, ARG) \\
\hline & 5 & $\begin{array}{l}\text { Licenciatura en Bibliotecología y } \\
\text { Ciencias de la Información }\end{array}$ & (UNP, ARG) \\
\hline & 6 & $\begin{array}{l}\text { Profesorado en Bibliotecología y } \\
\text { Ciencias de la Información }\end{array}$ & (UNP, ARG) \\
\hline & 7 & $\begin{array}{l}\text { Licenciatura en Bibliotecología y } \\
\text { Ciencias de la Información }\end{array}$ & (UMS, BOL) \\
\hline & 8 & $\begin{array}{l}\text { Gestión de Información, Bibliotecas } \\
\text { y Archivos }\end{array}$ & $(\mathrm{UAH}, \mathrm{CHL})$ \\
\hline & 9 & Bibliotecología & (UPA, CHL) \\
\hline & 10 & Biblioteconomía & (UTM, CHL) \\
\hline & 11 & $\begin{array}{l}\text { Ciencia de la Información- } \\
\text { Bibliotecología }\end{array}$ & (PUJ, COL) \\
\hline & 12 & Ciencia de la Información & (UQ, COL) \\
\hline & 13 & $\begin{array}{l}\text { Sistemas de Información y } \\
\text { Documentación }\end{array}$ & (US, COL) \\
\hline & 14 & $\begin{array}{l}\text { Bibliotecología y Ciencias de la } \\
\text { Información }\end{array}$ & (PUCP, PER) \\
\hline & 15 & $\begin{array}{l}\text { Bibliotecología y Ciencias de la } \\
\text { Información }\end{array}$ & (UNMSM,PER) \\
\hline & 16 & Licenciatura en Bibliotecología & (UCV, VEN) \\
\hline & 17 & Bibliotecología y Archivología & (UZ, VEN) \\
\hline \multirow[t]{3}{*}{$4-7$} & 1 & Biblioteconomía & (UB, BRA) \\
\hline & 2 & Biblioteconomía & (UFB, BRA) \\
\hline & 3 & Biblioteconomía & (UFP, BRA) \\
\hline 4.5 & 1 & Biblioteconomía & (UCSC, CHL) \\
\hline
\end{tabular}




\begin{tabular}{llll}
\hline 2 & $\begin{array}{l}\text { Ciencia de la Información y } \\
\text { Bibliotecología }\end{array}$ & (INPAHU, COL) \\
\cline { 2 - 4 } 3 & $\begin{array}{l}\text { Licenciatura en Ciencia de la } \\
\text { Información }\end{array}$ & (UNA, PAR) \\
\hline 1 & $\begin{array}{l}\text { Bachiller Superior en } \\
\text { Bibliotecología y Ciencias de la } \\
\text { Información }\end{array}$ & (UMS, BOL) \\
\hline 2 & Biblioteconomía & (UESPSP, \\
\hline 3 & Biblioteconomía & (URA, BRA) \\
\hline 4 & Biblioteconomía & (UFDRGS, \\
& BRA) \\
\hline 5 & Biblioteconomía & (UFSC, BRA) \\
\hline 6 & Bibliotecología & (UA, COL) \\
\hline 7 & $\begin{array}{l}\text { Historia con mención en } \\
\text { Archivología y Bibliotecología }\end{array}$ & (PUCE, ECU) \\
\hline 8 & Archivología y Bibliotecología & (UG, ECU) \\
\hline 9 & Bibliotecología & (UR, URU) \\
\hline 1 & Diplomatura en Bibliotecología & (UBA, ARG) \\
\hline 2 & Bibliotecología & (UNC, ARG) \\
\hline 3 & $\begin{array}{l}\text { Técnico Superior en Bibliotecología } \\
\text { y Ciencias de la Información }\end{array}$ & (UMSA, BOL) \\
\hline
\end{tabular}

Tabla III. Tiempo de duración de algunos programas de formación bibliotecológica en América del Sur

\subsection{Tendencias en la formación bibliotecológica} de algunas universidades de América del Sur

Las tendencias de formación bibliotecológica que más se destacan en los países de América del Sur, se enfocan en el desarrollo de los contenidos tradicionales de la bibliotecología, entre los que se señalan a la gestión de la información, los procesos de transferencia de la información, los servicios de información, la definición de políticas de información y por supuesto el uso y aplicación de las tecnologías de la información.

Se observan también, temáticas que marcan el enfoque de la formación, como es el caso de universidades argentinas que incluyen dentro de sus perfiles, énfasis en áreas como la docencia, la investigación, la tasación de colecciones bibliográficas documentales, peritajes referidos a la antigüedad y autenticidad y demás propiedades de determinadas fuentes de información, al igual que el desempeño interdisciplinario en el campo jurídico desde la perspectivas de los temas de la bibliotecología y las ciencias de la información, entre otros asuntos.

Algunas universidades de países como Brasil, Chile y Colombia, enfocan en sus contenidos a la biblioteca como institución social que contribuya con el desarrollo del país, y al profesional, como uno de los principales agentes de transferencia de información y de transformación social. También se observa que incluyen en sus perfiles el preparar profesionales con una visión crítica de la sociedad y con la capacidad de desarrollar una postura ética y un diálogo intercultural con la misma. Otros perfiles se manifiestan a la luz del desarrollo de competencias y ejes curriculares específicos al ámbito de la bibliotecología, como se aprecia en la propuesta de la Universidad Central de Venezuela.

Se logran identificar otras tendencias, pero que no se manifiestan por una mayoría de universidades, tales como la inclusión de contenidos enfocados a la investigación histórica, la asesoría a la producción editorial, la curaduría en museos, la conservación y difusión de los valores regionales, nacionales e internacionales, entre otros.

\subsection{Campos de desempeño más destacados}

Al aproximarse a los perfiles de formación bibliotecológica, también se identifican ambientes de desempeño profesional, unos tradicionales y otros que surgen como producto del advenimiento de nuevos paradigmas en cuanto a la gestión del conocimiento y de la información, así como al desarrollo tecnológico de las últimas décadas.

\subsubsection{Campos de desempeño profesional tradi- cional}

Todos los tipos de bibliotecas, centros de información y documentación, bancos, museos, centros culturales, hemerotecas, agencias de publicidad, despachos de abogados, editoras, periódicos, revistas, productoras de videos, colegios y universidades, archivos públicos y privados, empresas de ingeniería, centros de información, empresas comerciales, industriales y de servicios, organismos públicos de administración directa e indirecta, organizaciones gubernamentales, científicas, culturales, centros de investigación, librerías, ludotecas, centros de información empresarial, instituciones culturales, notarías, casas de la cultura, salas de exposiciones y de arte, organizaciones no gubernamentales, empresas binacionales, clubes sociales, hospitales, archivos de prensa, asesorías profesionales, entre otros.

\subsubsection{Nuevos campos de desempeño profesio-} nal

Bibliotecas virtuales, bases de datos, centros de medios, redes de telecomunicaciones, centros de recursos para el aprendizaje, consultorías en el manejo de la información, Centros e Institutos de Investigación, bibliotecas, videotecas, pinacotecas, servicios de consultoría, sitios de Internet, oficinas de consultoría en gestión del cono- 
cimiento, oficinas de diseño de servicios y productos de información, plataformas web, centros especializados en vigilancia tecnológica, Sistemas de información on line, empresas distribuidoras de productos de la información, empresas administradoras de recursos digitales y de sistemas integrados de información para organizaciones, empresas de servicios de la información.

\section{Conclusiones}

La formación bibliotecológica, se constituye en un campo de investigación que cobra mayor interés, debido al proceso de desarrollo por el que está atravesando, gracias a la influencia de factores como la globalización, el advenimiento de la sociedad de la información, el desarrollo de las TIC y las nuevas propuestas de desempeño profesional que se manifiestan como producto de la sociedad informatizada de hoy.

La educación bibliotecológica del siglo XXI, encara importantes retos, debido a las nuevas dimensiones de desempeño profesional que se han gestado como consecuencia del desarrollo tecnológico y el fortalecimiento de la Sociedad de la Información y del Conocimiento. En tal sentido, el perfil del profesional de la bibliotecología, se manifiesta como objeto de revisión y actualización, acorde con las nuevas tendencias que marca la sociedad actual.

Luego de hacer una aproximación a los perfiles de formación bibliotecológica de algunas universidades de América del Sur, se aprecia diversos niveles de formación, así como de titulación. Se identifican diferentes denominaciones en los procesos de formación en los que la bibliotecología, ya no se oferta como un tópico exclusivo. Se observan además, otras denominaciones más acordes con los nuevos paradigmas del desarrollo del ámbito de la información.

No obstante, generarse nuevas dimensiones del desempeño profesional bibliotecológico, se observa en los perfiles de formación de América del Sur, el desarrollo de actividades tradicionales, que son complementadas y optimizadas con la aplicación de las TIC. También se aprecia como algunas unidades académicas, manifiestan fuertes tendencias hacia el desarrollo de habilidades de orden social, comunicativo, tecnológico, investigativo, docente, gerencial, cultural, entre otras.

\section{Referencias}

Escalona Ríos, Lina. (Coordinadora) (2008, p. 8-9). La educación bibliotecológica en Iberoamérica // Escalona Ríos, Lina, (Coordinadora) (2008). Flexibilidad curricular en la educación bibliotecológica. México : UNAM, Centro Universitario de Investigaciones Bibliotecológicas, 2008. ISBN: 978-607-02-0769-3 p. 1-24

European Council of Information Associatins (ECIA) (Autor); Tejada Artigas, Carlos, (traductor) (2004). Euroreferencial en información y documentación : vol. I competencias y aptitudes de los profesionales europeos de información y documentación. Madrid : SEDIC, 2004. ISBN: 84-609-3634-1 $127 \mathrm{p}$.

Hawes, Barrio, Gustavo; Corvalán, Vásquez., Oscar (autores) (2006, p,9). Aplicación del enfoque de competencias en la construcción curricular de la Universidad de Talca, Chile. // Revista Iberoamericana de Educación, Dialnet. ISSN: 1681-56531.40: 1 (2006) p. 1-17

http://dialnet.unirioja.es/servlet/listaarticulos?tipo busqu eda $=E J E M P L A R \&$ revista_busqueda $=7466 \&$ clave_busqu eda=141881 (Fecha de consulta, julio 16 de 2012)

Martínez Rider, Rosa María; Chacón Escobar, Rosa Elba (Autoras) (2010, p. 44). Consideraciones en la estructuración de un perfil del bibliotecario Iberoamericano // Escalona Ríos, Lina, (Coordinadora) (2010). Las competencias en el perfil bibliotecológico en América Latina. México: UNAM, Centro Universitario de Investigaciones Bibliotecológicas, 2010. ISBN: 978-607-02-899-7 p.4355

Rodríguez Salas, Karla (Autora) (2009, p. 379). Perfil del bibliotecólogo: despejando la incertidumbre. // Martínez Arellano, Filiberto Felipe; Calva González, Juan José (Compiladores) (2009). Memoria del XXVI Coloquio de Investigación Bibliotecológica y sobre la Información., 1, 2, 3 de octubre de 2008. México : UNAM, Centro Universitario de Investigaciones Bibliotecológicas, 2009. ISBN: $978-607-02-0579-8$ p. 377-393

Enviado: 2012-08-22.

Aceptado: 2012-09-15.

\section{Apéndice}

\begin{tabular}{lll}
\hline País & Universidad & Programas académicos \\
\hline Argentina & Universidad de Buenos Aires, Facultad de Filosofía y Letras & Diplomatura en Bibliotecología \\
\cline { 3 - 3 } & & $\begin{array}{l}\text { Licenciatura en Bibliotecología y Ciencia de la } \\
\text { Información }\end{array}$ \\
\cline { 3 - 3 } & $\begin{array}{l}\text { Profesorado en Bibliotecología y Ciencia de la } \\
\text { Información. }\end{array}$ \\
\cline { 2 - 3 } & $\begin{array}{l}\text { Universidad Nacional de Córdoba, Facultad de Filosofía y } \\
\text { Humanidades, Escuela de Bibliotecología }\end{array}$ & Bibliotecología \\
\hline
\end{tabular}




\begin{tabular}{|c|c|c|c|}
\hline & & Licenciado en Bibliotecología y Documentación & 5 años \\
\hline & Universidad Nacional del Nordeste, Facultad de Humanidades & $\begin{array}{l}\text { Licenciatura en Ciencias de la Información (Con } \\
\text { orientación en bibliotecología o archivística) }\end{array}$ & 5 años \\
\hline & \multirow[t]{2}{*}{$\begin{array}{l}\text { Universidad Nacional de la Plata, Facultad de Humanidades y } \\
\text { Ciencias de la Educación, Departamento de Bibliotecología }\end{array}$} & $\begin{array}{l}\text { Licenciatura en Bibliotecología y Ciencias de la } \\
\text { Información }\end{array}$ & 5 años \\
\hline & & $\begin{array}{l}\text { Profesorado en Bibliotecología y Ciencias de la } \\
\text { Información }\end{array}$ & 5 años \\
\hline \multirow[t]{3}{*}{ Bolivia } & \multirow[t]{3}{*}{$\begin{array}{l}\text { Universidad Mayor de San Andrés, Facultad de Humanidades y } \\
\text { Ciencias de la Educación }\end{array}$} & $\begin{array}{l}\text { Técnico Superior en Bibliotecología y Ciencias } \\
\text { de la Información }\end{array}$ & 3 años \\
\hline & & $\begin{array}{l}\text { Bachiller Superior en Bibliotecología y Ciencias } \\
\text { de la Información }\end{array}$ & 4 años \\
\hline & & $\begin{array}{l}\text { Licenciatura en Bibliotecología y Ciencias de la } \\
\text { Información }\end{array}$ & 5 años \\
\hline \multirow[t]{7}{*}{ Brasil } & $\begin{array}{l}\text { Universidad de Brasilia, Facultad de Ciencia de la Información, } \\
\text { Departamento de Ciencia de la Información y la Documentación }\end{array}$ & Biblioteconomía & $\begin{array}{l}4 \text { a } 7 \\
\text { años }\end{array}$ \\
\hline & $\begin{array}{l}\text { Universidad Escuela de Sociología Política de Sao Paulo, Facultad de } \\
\text { Biblioteconomía y Ciencias de la Información }\end{array}$ & Biblioteconomía & 4 años \\
\hline & Universidad Federal de Bahía, Instituto de Ciencia de la Información & Biblioteconomía y Documentación & $\begin{array}{l}4 \text { a } 7 \\
\text { años }\end{array}$ \\
\hline & $\begin{array}{l}\text { Universidad Federal de Goias, Facultad de Comunicación y } \\
\text { Biblioteconomía }\end{array}$ & Biblioteconomía & 4 años \\
\hline & $\begin{array}{l}\text { Universidad Federal de Pernambuco, Centro de Artes y } \\
\text { Comunicación, Departamento de Ciencia de la Información }\end{array}$ & Biblioteconomía & $\begin{array}{l}4 \text { a } 7 \\
\text { años }\end{array}$ \\
\hline & $\begin{array}{l}\text { Universidad Federal de Río Grande Do Sur, Facultad de } \\
\text { Biblioteconomía y Comunicación }\end{array}$ & Biblioteconomía & 4 años \\
\hline & $\begin{array}{l}\text { Universidad Federal de Santa Catarina, Centro de Ciencias de } \\
\text { Educación, Departamento de Ciencia de la Información }\end{array}$ & Biblioteconomía & 4 años \\
\hline \multirow[t]{4}{*}{ Chile } & Universidad Alberto Hurtado, Facultad de Economía y Negocios & Gestión de Información, Bibliotecas y Archivos & 5 años \\
\hline & $\begin{array}{l}\text { Universidad Católica de la Santísima Concepción, Facultad de } \\
\text { Comunicación, Historia y Ciencias Sociales }\end{array}$ & Biblioteconomía y Documentación & 4.5 años \\
\hline & Universidad de Playa Ancha, Facultad de Ciencias Sociales & Bibliotecología & 5 años \\
\hline & $\begin{array}{l}\text { Universidad Tecnológica Metropolitana del Estado de Chile, Facultad } \\
\text { de Administración y Economía }\end{array}$ & Biblioteconomía y Documentación & 5 años \\
\hline \multirow[t]{5}{*}{ Colombia } & $\begin{array}{l}\text { Fundación Univeritaria Inpahu, Facultad de Ingeniería y Tecnologías } \\
\text { de la Información }\end{array}$ & Ciencia de la Información y Bibliotecología & 4.5 años \\
\hline & $\begin{array}{l}\text { Pontificia Universidad Javeriana, Facultad de Comunicación y } \\
\text { Lenguaje }\end{array}$ & Ciencia de la Información-Bibliotecología & 5 años \\
\hline & Universidad de Antioquia, Escuela Interamericana de Bibliotecología & Bibliotecología & 4 años \\
\hline & $\begin{array}{l}\text { Universidad del Quindio, Facultad de Ciencias Humanas y Bellas } \\
\text { Artes }\end{array}$ & Ciencia de la Información & 5 años \\
\hline & Universidad de la Salle, Facultad de Ciencias Económicas y Sociales & Sistemas de Información y Documentación & 5 años \\
\hline \multirow[t]{2}{*}{ Ecuador } & $\begin{array}{l}\text { Pontificia Universidad Católica del Ecuador, Facultad de Ciencias } \\
\text { Humanas }\end{array}$ & $\begin{array}{l}\text { Historia con mención en Archivología y } \\
\text { Bibliotecología }\end{array}$ & 4 años \\
\hline & $\begin{array}{l}\text { Universidad de Guayaquil, Facultad de Filosofía, Letras y Ciencias de } \\
\text { la Educación, Escuela de Bibliotecología }\end{array}$ & Archivología y Bibliotecología & 4 años \\
\hline Paraguay & Universidad de Asunción, Facultad Politécnica & Licenciatura en Ciencias de la Información & 4.5 años \\
\hline \multirow[t]{2}{*}{ Perú } & $\begin{array}{l}\text { Pontificia Universidad Católica del Perú, Facultad de Letras y Ciencias } \\
\text { Humanas, Departamento de Humanidades }\end{array}$ & Bibliotecología y Ciencias de la Información & 5 años \\
\hline & $\begin{array}{l}\text { Universidad Nacional Mayor de San Marcos, Facultad de Letras y } \\
\text { Ciencias Humanas, Escuela de Bibliotecología y Ciencias de la } \\
\text { Información }\end{array}$ & Bibliotecología y Ciencias de la Información & 5 años \\
\hline Uruguay & $\begin{array}{l}\text { Universidad de la República, Escuela Universitaria de Bibliotecología } \\
\text { y Ciencias Afines }\end{array}$ & Bibliotecología & 4 años \\
\hline \multirow[t]{2}{*}{ Venezuela } & $\begin{array}{l}\text { Universidad Central de Venezuela, Facultad de Humanidades y } \\
\text { Educación, Escuela de Bibliotecología y Archivología }\end{array}$ & Licenciatura en Bibliotecología & 5 años \\
\hline & $\begin{array}{l}\text { Universidad del Zulia, Facultad de Humanidades y Educación, } \\
\text { Escuela de Bibliotecología y Archivología }\end{array}$ & Bibliotecología y Archivología & 5 años \\
\hline
\end{tabular}

Tabla I. Programas de formación bibliotecológica de algunas universidades de América del Sur 